\title{
Entonación del español en contacto con el otomí de San Ildefonso Tultepec: enunciados declarativos e interrogativos absolutos
}

\author{
Intonation of the Spanish Language in Contact with \\ the Otomi Language Spoken in San Ildefonso Tultepec: \\ Declarative and Absolute Interrogative Statements
}

\author{
Eva Patricia Velásquez Upegui \\ Universidad Autónoma de Querétaro \\ docling@uaq.mx
}

Resumen

El objetivo en este documento es presentar una primera descripción de los rasgos entonativos del español en contacto con el otomí hablado en San Ildefonso Tultepec. Se describen y comparan los enunciados declarativos e interrogativos producidos por hablantes bilingües, y se trazan las similitudes y diferencias con la variedad estándar del español mexicano (Dela-Mota, Martín Butragueño y Prieto, 2010). El estudio se inscribe dentro del modelo métrico autosegmental (AM) y se siguen las consideraciones del sistema de transcripción $S p$ ToBI, recuperadas de Estebas-Vilaplana y Prieto (2008). Los resultados indican similitudes entre los enunciados declarativos e interrogativos producidos por los hablantes bilingües, sin embargo, se presentan claras diferencias con relación a la variedad estándar.

Palabras Clave: entonación, declarativas, interrogativas, contacto, español-otomí

Abstract

The purpose of this document is to provide an initial description of the intonation features of the Spanish language in contact with the Otomi language spoken in San Ildefonso Tultepec. The declarative and interrogative statements used by bilingual speakers are described and compared, and similarities and differences regarding the Mexican Spanish Standard Variety are traced (De-la-Mota, Martín Butragueño and Prieto, 2010). The study lies within the autosegmental (AM) metrical model and follows the considerations of the Sp-ToBI transcription system, set forth by Estebas-Vilaplana and Prieto (2008). The results show similarities among the declarative and interrogative statements used by bilingual speakers. However, there are clear differences regarding the standard variety.

KEYwORDS: intonation, declarative, interrogative, contact, Spanish-Otomi 


\section{Introducción}

El estudio de la lengua en la actualidad supone directa o indirectamente dar cuenta de las situaciones de contacto, debido a las condiciones sociales de migración (por motivos diversos) y de movilidad, que siguen siendo una constante en la historia de la formación de las sociedades y, por ende, de las lenguas. Por esta razón, resulta indispensable describir las características lingüísticas de variedades en contacto, en la medida en que su estudio contribuye a la comprensión de la lengua en uso, es decir, desde una perspectiva más realista en los contextos actuales de multilingüismo. Este es el caso de México y de otros países centro y suramericanos (Guatemala, Colombia, Perú, Paraguay, entre otros), donde el español convive en contacto frecuente con lenguas originarias (Chamoreau, 2013; Inali, 2008; Zimmermann, 2001).

En este sentido, se propone una descripción prosódica inicial, que considera la entonación de enunciados declarativos e interrogativos absolutos en el español en contacto con el otomí hablado en San Ildefonso Tultepec, de la zona de Amealco de Bonfil y, en principio, reconocer si estas características entonativas se diferencian del español de hablantes monolingües de la variedad metropolitana de la Ciudad de México (De-la-Mota, Martín Butragueño y Prieto, 2010). Para ello se parte de una exploración fonética de los enunciados, que tiene como base el modelo métrico autosegmental (AM), además se siguen las consideraciones del sistema de transcripción Sp-ToBI (Tones and Break Indices) (Estebas-Vilaplana y Prieto, 2008).

En adelante se presentan brevemente algunas consideraciones teóricas que contemplan la lengua otomí y el español en contacto con lenguas originarias; a continuación se expone la metodología empleada; posteriormente, se presentan los resultados, a manera de comparación entre los enunciados declarativos e interrogativos absolutos $y$, por último, se ofrecen unas conclusiones preliminares. 


\section{Consideraciones teóricas}

\subsection{La lengua otomí}

Las lenguas otomíes pertenecen al grupo oto-pameano de la familia lingüística oto-mangue conformada por los grupos tlapaneco-mangue, popoloca-zapoteco, amuzgo miztecano, otopame y chinanteco. Conforma el grupo otopame dentro de la rama otomiana (otomí, mazahua, matlatzinca y ocuilteco) que lo integra junto con la pameana (pame y chichimeca).

De acuerdo con la información del Catálogo de lenguas indígenas consignadas por el Instituto Nacional de Lenguas Indígenas (Inali) para el 2008, como agrupación lingüística, el otomí se encuentra conformado por las siguientes nueve variantes lingüísticas: 1) otomí de la Sierra, 2) otomí bajo del noroeste, 3) otomí del oeste, 4) otomí del oeste del Valle del Mezquital, 5) otomí del Valle del Mezquital, 6) otomí de Ixtenco, 7) otomí de Tilapa o del sur, 8) otomí del noroeste, y 9) otomí del centro (Nava, 2010). De acuerdo con Palancar (2009), la comunidad otomí de San Ildefonso Tultepec proviene del estado de Hidalgo, lo que la relaciona con la variante 1 , hablada en los estados de Hidalgo, Puebla y Veracruz.

Con relación a los rasgos suprasegmentales, la lengua otomí cuenta con tres tonos léxicos: alto, bajo y ascendente. Todas las raíces léxicas y los clíticos reciben un tono inherente, que puede modificarse prosódicamente de acuerdo con reglas morfofonológicas. Palancar menciona que "a pesar de la existencia del tono, se tienen relativamente pocos pares léxicos mínimos” (2009, p. 11).

Guerrero (2015) hace una revisión del tono a nivel léxico a partir de evidencia acústica y encuentra que en la variedad del otomí de Santiago Mexquititlán, la aparición más frecuente es la formación de palabra prototípica que posee una configuración tonal de bajo alto ('B.A) y alto alto ('A.A) con seis combinaciones posibles. $\mathrm{El}$ autor menciona que hay una interacción entre los patrones morfológicos y acentuales de la lengua, y que el acento tiene una función morfológica demarcativa debido a que cae obligatoriamente en la raíz. Por estas razones, Guerrero considera que el otomí es una lengua tono-acentual. 
A pesar de que no existe una descripción previa de la entonación del otomí de San Ildefonso Tultepec, se menciona que, a nivel oracional, hay un acento entonacional largo y fuerte que se encuentra a nivel de frase (Guerrero, 2015; Palancar, 2009). Hekking y Andrés de Jesús (1984), señalan, a propósito de la variedad de Santiago Mexquititlán, que la oración afirmativa se opone a los otros tipos principales de oraciones, por no estar marcada mediante un elemento sintáctico o morfológico, mientras que la oración interrogativa inicia con la partícula $h a^{1}$ y su entonación es ascendente, es decir, presenta una elevación de la frecuencia sobre la última sílaba de la oración.

\subsection{Entonación del español en contacto con lenguas originarias}

Actualmente existe cada vez más interés en la descripción prosódica del español en contacto con diferentes lenguas, dando lugar a múltiples estudios (Alvord, 2006; Colantoni, 2011; Dorta, 2019; Elordieta, 2003; Robles-Puente, 2012; Simonet, 2011) que han logrado explicar que, en efecto, es posible hablar de modificaciones en la estructura prosódica del español derivadas, justamente, del contacto lingüístico.

Entre los estudios del español en contacto con lenguas originarias, se encuentra el trabajo de O'Rourke (2012), con hablantes bilingües español-quechua del Cuzco y hablantes monolingües del español de Lima. La autora analiza la realización prosódica de foco amplio y contrastivo de acuerdo con el alineamiento y la altura tonal, y concluye que, en ambos tipos de foco, el pico tonal se realiza dentro de la sílaba acentuada, además, los hablantes de Lima emplean una F0 más alta y un rango tonal más amplio para el foco contrastivo en comparación con el amplio, mientras que los hablantes bilingües presentaron el patrón contrario.

\footnotetext{
${ }^{1}$ Palancar (2009) señala esta misma característica para las interrogativas absolutas del otomí de San Ildefonso Tultepec.
} 
Muntendam (2012) analiza la estructura de la información y la entonación en el español andino hablado por bilingües españolquechua, también del estado de Cuzco. La autora encuentra que la duración, la altura de la F0 y el alineamiento del pico no son usados consistentemente para diferenciar el foco amplio de foco contrastivo, a diferencia de lo que sucede con otras variedades del español. En línea con el anterior trabajo, van Rijswijk y Muntendam (2014) analizan la prosodia de foco amplio y de foco contrastivo en bilingües quechua-español del Cuzco. Los autores descubren que los hablantes bilingües emplean estrategias prosódicas diferentes al español de los monolingües para marcar el foco, como el hecho de no utilizar el prealineamiento para hacer el contraste entre un foco amplio y estrecho, y la presencia de una F0 alta asociada al foco amplio. Muntendam y Torreira (2016) reportan hallazgos similares al comparar focos amplios y focos estrechos en hablantes monolingües de español peninsular y de quechua, y bilingües español-quechua, es decir, los autores encuentran configuraciones entonativas en el español hablado por los bilingües que se presentan en el quechua pero no fueron halladas en la variedad peninsular.

Por su parte, Dankel y Soto (2018), analizan las enumeraciones en quechua, en el español andino y en el español peninsular, y hallaron una fuerte coincidencia entre los patrones prosódicos del quechua y el español andino que divergen del español peninsular. Ruiz, Ulloa y Chihuaicura (2019) estudian los enunciados declarativos en hablantes monolingües del español de Chile, y hablantes bilingües español-mapundungun. Los autores descubrieron una mayor presencia de tonos nucleares y junturas terminales ascendentes en los tonemas de ambos grupos de hablantes, aunque los movimientos ascendentes fueron más comunes en hablantes que habitaban zonas rurales alejadas del núcleo urbano.

Henriksen y Fafulas (2017), analizan el timing prosódico en el español hablado por monolingües de Iquitos (Perú) y bilingües español-yagua. Los datos analizados revelan que los hablantes bilingües tienen valores temporales similares a los del español con independencia de la lengua dominante.

De otro lado, Michnowicz y Barnes (2013) estudian el alineamiento del pico tonal en el segmento prenuclear del español 
de Yucatán con hablantes bilingües y monolingües. Los autores documentan una mayor proporción en la realización de picos tempranos en ambos grupos de hablantes. Con este mismo objetivo, Barnes y Michnowicz (2013) analizan enunciados declarativos en hablantes bilingües veneto-español o español chipilo (Puebla, México), y encuentran un amplio porcentaje de picos prealineados en comparación con otras variedades del español que no están en situaciones de contacto.

Con relación al prealineamiento del pico tonal, Braid (2015) analiza las sílabas prenucleares en hablantes monolingües de Quetzaltenango y bilingües español-K'ichee' de Cantel y Nahualá, en Guatemala. El autor explica que el prealineamiento del pico se presenta en los diferentes grupos, sin embargo, señala que hay diferencias significativas en los hablantes bilingües con mayor dominancia en la lengua originaria. Velázquez Patiño (2016) compara la entonación en hablantes de náhuatl (región de la huasteca veracruzana) como L1, al producir enunciados interrogativos absolutos y pronominales en náhuatl y español, y en hablantes monolingües de español de la misma región. El autor indica que estos enunciados presentan los mismos patrones entonativos en los diferentes grupos de hablantes.

Por su parte, Uth y Gutiérrez-Bravo (2020) estudian el contacto lingüístico entre el maya y el español yucateco en la realización entonativa de foco contrastivo y la prosodia de los préstamos del español. Los autores explican que en ambas variedades la realización del elemento más prominente se encuentra en la parte más izquierda de la frase entonativa, a diferencia de lo que sucede en el español estándar de la Ciudad de México.

\section{Metodología}

\subsection{Instrumentos}

En general, los datos colectados son enunciados controlados e inducidos a partir de una serie de instrumentos. ${ }^{2}$ En primer

\footnotetext{
${ }^{2}$ No se han incluido datos de habla espontánea.
} 
lugar, se contó con una serie de 20 imágenes fotográficas para que los participantes respondieran a la pregunta ¿Qué ve en la imagen?, de modo que produjeran enunciados declarativos de foco amplio o también llamado foco presentativo o informativo, es decir, el foco o realce coincide con toda la información contenida en el enunciado. ${ }^{3}$ Se produjeron enunciados del tipo Los niños están corriendo o Unos niños corriendo (figura 1).

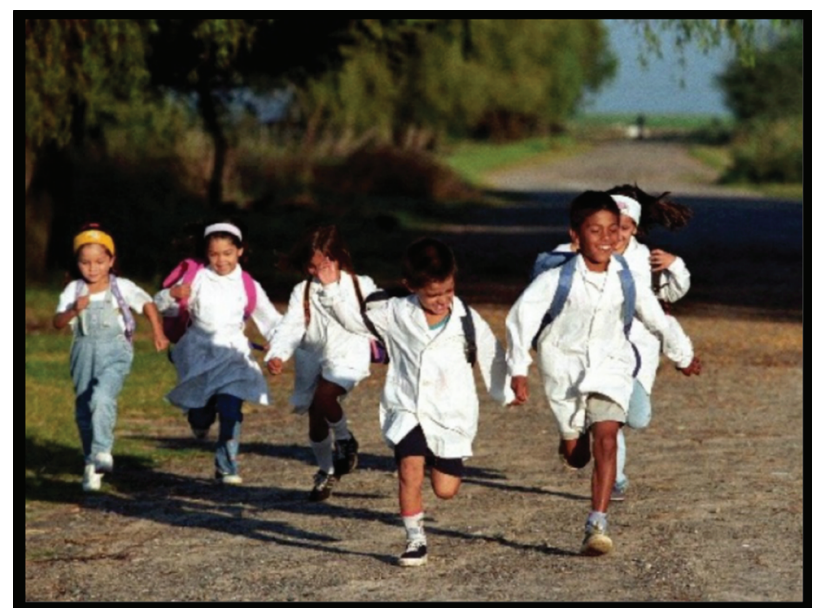

Figura 1. Imagen para la producción de enunciados. ${ }^{4}$ (Kokcmann, s/f)

Para reunir los enunciados interrogativos absolutos se empleó la técnica de completamiento discursivo (Prieto y Roseano, 2010). Se construyeron contextos discursivos para que el hablante produjera el enunciado interrogativo esperado como en (1).

1) Ves que tu amiga viene caminando y quieres saber si viene del mandado.

Pregúntaselo

Enunciado esperado: ¿Vienes del mandado?

\footnotetext{
${ }^{3}$ Los focos amplios, por lo regular, proporcionan respuestas a preguntas en las que no se identifica o destaca algún elemento en particular (Gutiérrez-Bravo, 2008).

${ }^{4}$ Las imágenes son una serie de fotografías documentales de retratos a color, con un diafragma abierto sin descontextualizar el entorno de la persona(s) retratada(s).
} 
En total se colectaron 20 enunciados declarativos y 20 interrogativos absolutos por cada hablante. Los enunciados fueron grabados en las viviendas de los participantes, de modo que algunos datos fueron dejados de lado por contener demasiado ruido del ambiente. Además, se analizaron exclusivamente enunciados terminados en palabra paroxítona para mantener los mismos criterios en la descripción. Se les pidió a los informantes que produjeran los enunciados en dos ocasiones, de modo que la primera sirviera de entrenamiento, en el análisis sólo fueron consideradas las segundas emisiones en todos los casos. ${ }^{5}$

\subsection{Participantes}

Se grabaron a cuatro mujeres bilingües de San Ildefonso Tultepec (MBLSI), con el otomí como lengua materna. Todas las participantes son mayores de 44 años de edad, con nivel de instrucción bajo (primaria). ${ }^{6}$ En general, se dedican a las labores del hogar, con empleos eventuales en la ciudad de Querétaro. Las participantes fueron grabadas en sus casas ubicadas en el municipio de Amealco, en la comunidad del Bothé (cuadro 1).

\begin{tabular}{|c|c|c|c|c|}
\hline Hablante & Edad & Escolaridad & Oficio & $\begin{array}{c}\text { Edad de adquisición } \\
\text { del español }\end{array}$ \\
\hline M1 BLSI & 66 & Primaria & $\begin{array}{c}\text { Ama de casa- } \\
\text { Artesana }\end{array}$ & Otomí-Español (13 años) \\
\hline M2 BL SI & 46 & Primaria & Ama de casa & Otomí-Español (7 años) \\
\hline M3 BL SI & 45 & Primaria & Ama de casa & Otomí-Español (7 años) \\
\hline M4 BL SI & 45 & Primaria & Ama de casa & Otomí-Español (7 años) \\
\hline
\end{tabular}

Cuadro 1. Hablantes bilingües otomí-español

\footnotetext{
${ }^{5}$ Los hablantes bilingües produjeron los enunciados tanto en español como en otomí. ${ }^{6}$ Se eligieron las participantes de esta edad debido a que son pocas las personas menores que hablan la lengua en la comunidad, y además, no aprendieron el otomí como lengua materna. Con relación al nivel de estudios, las participantes entrevistadas no cuentan con estudios posteriores a la educación primaria.
} 
Para determinar el grado de dominancia de las lenguas, las participantes diligenciaron el cuestionario Bilingual Language Profile (BLP), que permite establecer una valoración cuantitativa a partir del perfil lingüístico de los hablantes bilingües con base en su historial lingüístico, el uso de las lenguas, y las actitudes y proficiencia (Birdsong, Gertken, y Amengual, 2012) (cuadro 2).

\begin{tabular}{|c|c|c|c|c|}
\hline Hablante & Edad & Español & Otomí & Diferencia \\
\hline M1 BL SI & 66 & 131.672 & 122.134 & 10 Español \\
\hline M2 BL SI & 46 & 113.508 & 135.304 & 22 Otomí \\
\hline M3 BL SI & 45 & 152.926 & 115.594 & 37 Español \\
\hline M4 BL SI & 45 & 103.79 & 144.2 & 40 Otomí \\
\hline
\end{tabular}

Cuadro 2. Dominancia de lenguas en hablantes bilingües otomí-español

De acuerdo con los resultados arrojados por el BLP (cuadro 2), las hablantes presentan similar grado de dominancia de ambas lenguas y solo en las hablantes M2 y M4, se encuentra el otomí por encima del español. Con relación a las secciones del cuestionario, en las respuestas concernientes al historial lingüístico, el uso de lenguas y la proficiencia, se encuentra que en todos los casos el español está por encima del otomí, sin embargo, en el apartado de actitudes, que involucra preguntas de identidad cultural con la lengua, en las cuatro hablantes, el otomí se encuentra por encima del español.

\subsection{Procedimiento}

Se parte del enfoque del modelo métrico autosegmental (AM) ( Pierrehumbert, 1980; Ladd, 1996; Hualde, 2003; Prieto, 2003; Gussenhoven, 2004) que está diseñado para dar cuenta de los elementos contrastivos del sistema entonativo. Este abordaje permite la descripción de los contornos melódicos presentes en los enunciados objeto de estudio. Adicionalmente, el modelo permite identificar segmentos fonológicos, que son etiquetados 
con el sistema de notación para el español Sp_ToBI (EstebasVilaplana y Prieto, 2008). ${ }^{7}$

El análisis acústico se llevó a cabo con ayuda del software Praat (Boersma y Weenink, 1992-2019), para realizar la medición en los diferentes puntos del enunciado. Inicialmente, se restauró el pitch en los casos donde la lectura de la F0 se encontraba alterada por el ruido del ambiente, por el efecto de voz laringizada en el contorno melódico o por una caída del tono generada por efectos microprosódicos.

Se calculó el promedio de la F0 de las vocales en cada sílaba del tonema, y se establecieron las mediciones en semitonos (st.). Se extrajo la diferencia entre los valores obtenidos en las sílabas del tonema. Asimismo, se establecieron los promedios y la diferencia entre el inicio y la primera sílaba tónica. Se calculó el tono de juntura inicial con respecto al tono básico ${ }^{8}$ de las informantes (Ávila, 2003). Se midió la declinación en el cuerpo del enunciado, a partir de la diferencia entre la primera sílaba tónica y la sílaba prenuclear y, por último, se construyó la base de datos con los valores normalizados a st. y las transcripciones fonéticas.

El etiquetado se efectuó teniendo en cuenta el movimiento de la curva tonal y la diferencia alcanzada en semitonos (st). De acuerdo con el criterio del umbral de percepción de 1.5 st. (Pamies, Fernández, Martínez-Celdrán, Ortega y Amorós, 2002; Murrieta, 2016 $)^{9}$ los movimientos menores a 1.5 st. se transcribieron con monotonos $\left(\mathrm{H}^{*}, \mathrm{~L}^{*}\right)$, y para los movimientos mayores a 1.5 st. se emplearon los bitonos $\left(\mathrm{L}+\mathrm{H}^{\star}, \mathrm{L}^{\star}+\mathrm{H}, \mathrm{L}+>\mathrm{H}^{*}\right.$, $\left.\mathrm{H}+\mathrm{L}^{\star}\right)$. Para los tonos de juntura terminal se emplearon los monotonos $\mathrm{L} \%, \mathrm{M} \%$ y $\mathrm{H} \%$.

\footnotetext{
${ }^{7}$ En Hualde y Prieto (2015) se han presentado modificaciones al etiquetado para hacerlo común y comparable con las diversas lenguas romances (Frota y Prieto, 2015), sin embargo, dados los intereses de esta investigación se ha decidido mantener la transcripción según el inventario previo.

${ }^{8}$ Promedio tonal de todas las mediciones de la F0 en la vocal al inicio de los enunciados, por cada participante.

${ }^{9}$ Rietveld y Gussenhoven (1985), con datos del holandés, encontraron que una diferencia de 1.5 st permite percibir diferencias en la F0.
} 


\section{Resultados}

Los resultados de la entonación en los enunciados declarativos e interrogativos absolutos de esta variedad en contacto se abordarán a partir de la comparación entre ambos grupos de enunciados. En principio, se muestran las diferencias asociadas al tono de juntura inicial, luego se aborda el segmento pretonemático y, posteriormente, se exponen los patrones encontrados en el tonema.

\subsection{El tono de juntura inicial: comparación entre enunciados declarativos e interrogativos absolutos}

Los enunciados declarativos al igual que los interrogativos absolutos presentan tanto inicios bajos $\% \mathrm{~L}$ como altos $\% \mathrm{H}$, es decir, por debajo y por encima del tono medio de los informantes. En los enunciados declarativos, 44 de los 80 enunciados en total, se realizan con un tono de juntura inicial bajo \%L, y 36 enunciados con tono alto $\% \mathrm{H}^{10}$ (cuadro 3). En los enunciados interrogativos, 48 de los enunciados presentan tonos de juntura inicial bajos \%L, mientras que los 32 enunciados restantes presentan junturas altas $\% \mathrm{H}$ (cuadro 3 ).

\begin{tabular}{|c|c|c|c|}
\hline Tonos de juntura inicial & L\% & H\% & $\begin{array}{c}\text { Total junturas } \\
\text { iniciales }\end{array}$ \\
\hline \multirow{2}{*}{ Enunciados Declarativos } & 44 & 36 & 80 \\
\cline { 2 - 4 } & $55.00 \%$ & $45.00 \%$ & $100.00 \%$ \\
\hline \multirow{2}{*}{$\begin{array}{c}\text { Enunciados } \\
\text { Interrogativos }\end{array}$} & 48 & 32 & 80 \\
\hline \multirow{2}{*}{ Total N } & $60.00 \%$ & $40.00 \%$ & $100.00 \%$ \\
\cline { 2 - 4 } & 92 & 68 & 160 \\
\hline
\end{tabular}

Cuadro 3. Tonos de juntura inicial enunciados declarativos e interrogativos absolutos

\footnotetext{
${ }^{10}$ La hablante M3 realizó un mayor número de junturas iniciales altas en los enunciados declarativos, este mismo comportamiento se reportó en los enunciados interrogativos emitidos por M3, en comparación con las demás participantes.
} 
Respecto al primer acento tonal se encuentra que para los enunciados declarativos el $62 \%$ de los datos presentaron un tono $\mathrm{H}^{*}$, seguido por $\mathrm{L}+\mathrm{H}^{*}$, con el $28.75 \%$; también se presentaron con menos ocurrencias los acentos tonales $\mathrm{L}+>\mathrm{H}^{\star}$ y $\mathrm{L}^{\star}$, que obtuvieron el 5\% y $3.75 \%$, respectivamente. ${ }^{11}$ Los enunciados interrogativos revelaron un comportamiento similar en cuanto a la realización de acentos tonales $\mathrm{H}^{*}$, que tuvieron una ocurrencia del $63.75 \%$, el $36.25 \%$ restante fue para el acento $\mathrm{L}+\mathrm{H}^{*}$.

\subsection{Declinación en el pretonema de los enunciados declarativos e interrogativos absolutos}

Para determinar la declinación en el pretonema o cuerpo del enunciado, se calculó la diferencia entre la altura de la primera sílaba tónica del enunciado y la sílaba prenuclear. Los resultados se agruparon de acuerdo con el umbral de percepción de 1.5 st., de este modo se clasificaron como descensos o ascensos los movimientos mayores a 1.5 st. según la dirección del movimiento, y sostenidos para los movimientos menores a 1.5 st. (cuadro 4).

\begin{tabular}{|c|c|c|c|c|}
\hline Pretonema & Descensos & Sostenidos & Ascensos & Total \\
\hline \multirow{2}{*}{\begin{tabular}{c} 
Enunciados declarativos \\
\cline { 2 - 5 }
\end{tabular}} & 28 & 35 & 17 & 80 \\
\hline $\begin{array}{c}\text { Enunciados interrogativos } \\
\text { absolutos }\end{array}$ & $45.00 \%$ & $43.75 \%$ & $21.25 \%$ & $100.00 \%$ \\
\cline { 2 - 5 } & $5.00 \%$ & $52.50 \%$ & $42.50 \%$ & $100.00 \%$ \\
\hline \multirow{2}{*}{ Total } & 32 & 77 & 51 & 160 \\
\cline { 2 - 5 } & $40.00 \%$ & $96.25 \%$ & $63.75 \%$ & $100.00 \%$ \\
\hline
\end{tabular}

Cuadro 4. Declinación en el pretonema en enunciados declarativos e interrogativos absolutos

\footnotetext{
${ }^{11}$ Particularmente en las hablantes, se observa que M1 presenta una mayor cantidad de acentos $\mathrm{L}+\mathrm{H}^{*}$, mientras que las demás participantes, M2, M3 y M4, presentaron más ocurrencias con el acento $\mathrm{H}^{\star}$. En este caso es probable que se trate de un efecto de las características particulares de la participante (cuadro 1).
} 
En ambos tipos de enunciados, el pretonema sostenido tiene una mayor frecuencia en comparación con el descenso y el ascenso, sin embargo, en los enunciados interrogativos el $42.5 \%$ de los datos reveló un trayecto ascendente a diferencia de lo que sucede con los enunciados declarativos, en los que aparece el descenso en segundo lugar de ocurrencia con el 35\% de los datos. Los enunciados interrogativos pueden presentar movimientos ascendentes en las sílabas tónicas del contorno prenuclear, aunque en los casos de finales anticadentes no exceden el ascenso final. ${ }^{12}$

Estos resultados difieren de la realización reportada por De-la-Mota, Martín Butragueño y Prieto (2010) para los enunciados declarativos en la Ciudad de México. Los autores documentan la presencia de picos desplazados en el segmento prenuclear $\mathrm{L}+>\mathrm{H}^{*}$, que fue realmente escaso en los datos revisados, pues las hablantes bilingües presentaron mayores sostenimientos o contornos relativamente planos. De igual manera, difieren con la realización de los enunciados interrogativos descrita por los autores, dado que refieren la presencia de tonos $\mathrm{L}^{*}+\mathrm{H}$ y $\mathrm{L}+>\mathrm{H}^{\star}$ que, aunque también se presentaron en las hablantes bilingües, la frecuencia fue considerablemente menor en comparación con la realización plana.

\subsection{El tonema en los enunciados declarativos e interrogativos absolutos}

En el tonema de los enunciados declarativos, es decir, en la última sílaba tónica del enunciado seguida de la sílaba postónica, se presenta con mayor recurrencia el contorno ascendente $\mathrm{L}+\mathrm{H}^{*},{ }^{13}$

\footnotetext{
${ }^{12}$ Las hablantes M3 y M4 presentaron el mayor número de ocurrencias en los descensos, por su parte, las hablantes M1 y M3, mostraron una mayor frecuencia de enunciados en la realización sostenida, lo que podría ser un efecto de la dominancia de lengua (cuadro 2). En los enunciados interrogativos, la hablante M1 presentó mayores ocurrencias en los ascensos, y menores, en los descensos. Las demás hablantes mostraron frecuencias similares en cada trayecto.

${ }^{13}$ Esta configuración del tono nuclear también se ha reportado en variedades del español mexicano como Cuapiaxtla (Mendoza, 2014), Tlachco y Michoacán (Coro-
} 
con final descendente L\%, y, en menor medida, el movimiento sostenido plano $\left(\mathrm{H}^{*}\right)^{14}$ que puede finalizar de manera sostenida M\% o en descenso L\%. ${ }^{15}$ Aunque con muy pocas ocurrencias también se presenta el bitono de frontera HL\%, es decir, un ascenso y descenso al finalizar el enunciado (cuadro 5). Lo que indica que, si bien hay una tendencia al ascenso nuclear al final del enunciado, las hablantes pueden optar por realizaciones completamente planas.

La realización prosódica de este tipo de enunciados en la Ciudad de México presenta algunas diferencias con relación a la encontrada en los hablantes bilingües. De-la-Mota, Martín Butragueño y Prieto (2010) reportaron dos realizaciones para el tonema en la Ciudad de México; la primera, considerada como la más frecuente, con un tono descendente en la sílaba nuclear $L^{*}$, más una juntura terminal baja $\mathrm{L} \% \mathrm{y}$, la segunda, encontrada en datos de habla semiespontánea, con un tono circunflejo $\mathrm{L}+\mathrm{H}^{*}$ seguido por un final descendente L\%. Esta última coincide con la configuración de las hablantes bilingües que hacen parte de este estudio.

\begin{tabular}{|c|c|c|c|c|c|}
\hline \multicolumn{2}{|c|}{ Tonema } & \multicolumn{3}{|c|}{ Juntura terminal } & \multirow{3}{*}{ 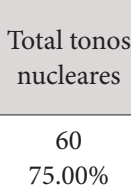 } \\
\hline & & HL\% & M\% & $\mathrm{L} \%$ & \\
\hline \multirow{2}{*}{$\begin{array}{c}\text { Sílaba } \\
\text { nuclear }\end{array}$} & $\mathrm{L}+\mathrm{H}^{*}$ & $\begin{array}{c}3 \\
3.75 \%\end{array}$ & $\begin{array}{c}24 \\
30.00 \%\end{array}$ & $\begin{array}{c}33 \\
41.25 \%\end{array}$ & \\
\hline & $\mathrm{H}^{*}$ & 0 & $\begin{array}{c}11 \\
13.75 \%\end{array}$ & $\begin{array}{c}9 \\
11.25 \%\end{array}$ & $\begin{array}{c}20 \\
25.00 \%\end{array}$ \\
\hline \multicolumn{2}{|c|}{$\begin{array}{c}\text { Total de tonos de juntura } \\
\text { terminal }\end{array}$} & $\begin{array}{c}3 \\
3.75 \%\end{array}$ & $\begin{array}{c}35 \\
43.75 \%\end{array}$ & $\begin{array}{c}42 \\
52.50 \%\end{array}$ & $\begin{array}{c}80 \\
100.00 \%\end{array}$ \\
\hline
\end{tabular}

Cuadro 5. Frecuencia de acentos nucleares y junturas terminales en enunciados declarativos

nado, 2014), La Paz (Gil, 2011) y Tuxtla (Mendoza, 2019a). Igualmente, Martín Butragueño $(2004,2006,2011)$ ha documentado esta realización circunfleja en el centro de México.

${ }^{14}$ En estos enunciados la hablante M3, a diferencia de las demás participantes, presentó mayores frecuencias con el acento nuclear $\mathrm{H}^{*}$.

${ }^{15}$ La hablante M1 tuvo mayor número de junturas terminales L\%. 
En el enunciado declarativo Los niños están corriendo, la sílaba nuclear -rrien- presenta el mayor ascenso de toda la emisión, mientras que el pretonema transcurre de manera relativamente plana (figura 2). Incluso en los enunciados con el tonema $\mathrm{H}^{*} \mathrm{~L} \%$, el pretonema mantiene una realización plana o sostenida. Por su parte, el pico en la sílaba nuclear se presenta antes de finalizar la sílaba para culminar con una juntura descendente.

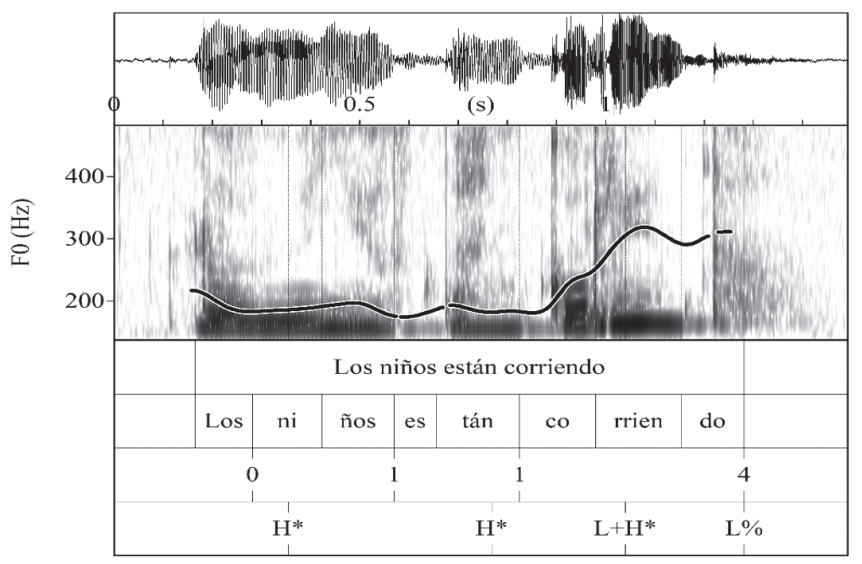

Figura 2. Enunciado declarativo. M2BLSI Los niños están corriendo

Los enunciados interrogativos absolutos se caracterizan por presentar en el tonema un movimiento ascendente en la sílaba nuclear $\mathrm{L}+\mathrm{H}^{* 16}$ acompañado por un final descendente L\% (Figura 3), son más escasos los finales del tipo M\% y $\mathrm{H} \%$. $\mathrm{Al}$ igual que en los enunciados declarativos, este patrón parece alternar con realizaciones más sostenidas $\mathrm{H}^{* 17}$ y finales igualmente sostenidos M\% (cuadro 6). Por su parte, en el español de la Ciudad de México, De-la-Mota, Martín Butragueño y Prieto (2010) registran un tono bajo $L^{*}$ con una juntura terminal ascendente- descendente HL\%, a diferencia de lo que sucede con los datos de este estudio.

\footnotetext{
${ }^{16}$ Esta configuración en el tonema de enunciados interrogativos también se documenta para el español de la Ciudad de México (Ávila, 2003), en Monterrey (Montellano, 2017) y Morelia (Mendoza, 2019b).

${ }^{17} \mathrm{El}$ tonema $\mathrm{H}^{\star} \mathrm{M} \%$ tuvo mayor frecuencia en los enunciados emitidos por M4.
} 


\begin{tabular}{|c|c|c|c|c|c|}
\hline \multicolumn{2}{|c|}{ Tonema } & \multicolumn{3}{|c|}{ Juntura terminal } & \multirow{3}{*}{$\begin{array}{c}\text { Total tonos } \\
\text { nucleares }\end{array}$} \\
\hline & & $\mathrm{H} \%$ & M\% & L\% & \\
\hline \multirow[t]{2}{*}{$\begin{array}{l}\text { Sílaba } \\
\text { nuclear }\end{array}$} & $\mathrm{L}+\mathrm{H}^{*}$ & $\begin{array}{c}3 \\
3.75 \%\end{array}$ & $\begin{array}{c}15 \\
18.75 \%\end{array}$ & $\begin{array}{c}46 \\
57.50 \%\end{array}$ & \\
\hline & $\mathrm{H}^{*}$ & 0 & $\begin{array}{c}16 \\
20.00 \%\end{array}$ & 0 & $\begin{array}{c}16 \\
20.00 \%\end{array}$ \\
\hline \multicolumn{2}{|c|}{$\begin{array}{l}\text { Total de tonos de } \\
\text { juntura terminal }\end{array}$} & $\begin{array}{c}3 \\
3.75 \%\end{array}$ & $\begin{array}{c}31 \\
38.75 \%\end{array}$ & $\begin{array}{c}46 \\
57.50 \%\end{array}$ & $\begin{array}{c}80 \\
100.00 \%\end{array}$ \\
\hline
\end{tabular}

Cuadro 6. Frecuencia de acentos nucleares y junturas terminales en enunciados interrogativos

El enunciado interrogativo ¿Los niños están corriendo? revela un movimiento ascendente en la sílaba nuclear -rrien-con el pico alineado al centro de la sílaba desde donde empieza el descenso (figura 3). El ascenso en la sílaba nuclear es mucho mayor en relación con el resto del enunciado que transcurre sin mayores inflexiones y su realización final concluye con el descenso.

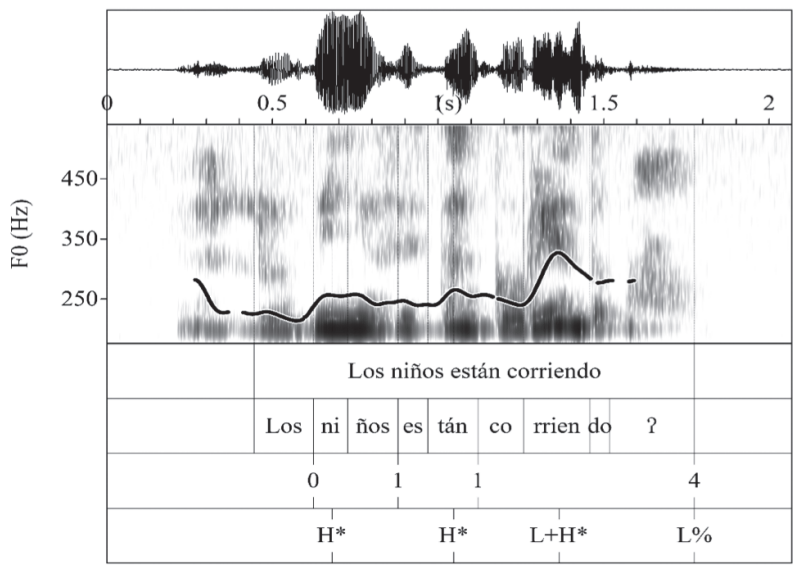

Figura 3. Enunciado interrogativo absoluto. M2 BL SI ¿Los niños están corriendo?

Dado que, en las hablantes bilingües, el patrón de mayor ocurrencia, tanto en los enunciados declarativos como en los interrogativos absolutos, presenta un movimiento ascendente en el tonema, en adelante el análisis se centrará en este grupo de datos. 
Un rasgo de bastante notoriedad que se da en los enunciados interrogativos y que no se encuentra en los enunciados declarativos de las hablantes bilingües, lo constituye la inserción de una unidad segmental, subsecuente al tono de juntura terminal. Se trata de un segmento caracterizado por un cierre laríngeo y una soltura aspirada que se presenta al finalizar el enunciado. ${ }^{18}$ Es muy probable que este segmento corresponda a un sonido glotal, ${ }^{19}$ puesto que presenta características como una duración de la detención de la fonación que alcanza en promedio $94 \mathrm{~ms}$. Este valor excede el promedio de 20 ms empleado por Frazier (2009) y Braid y Francisco (2012) para identificar los cierres glotales en lenguas mayas. De igual manera, el segmento glotal presenta ausencia de F0, o bien un notable descenso, y una disminución de la intensidad (de $16.1 \mathrm{~dB}$, en promedio) en comparación con el elemento adyacente. Además, en los casos en que los enunciados finalizan en vocal, una porción de este segmento adyacente se produce con voz glotalizada. ${ }^{20}$

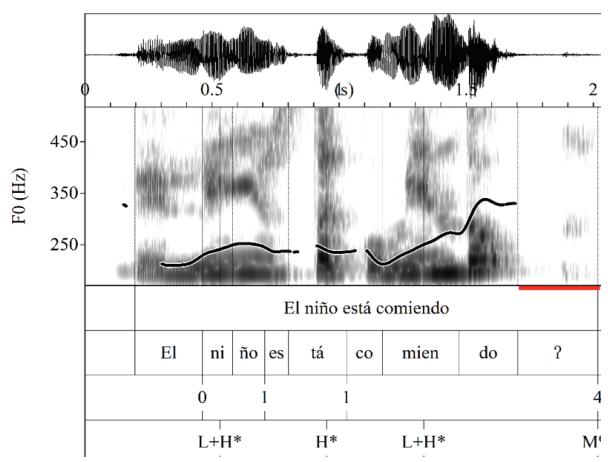

Figura 4. Enunciados interrogativos absolutos. M1BLSI ¿El niño está comiendo?

\footnotetext{
${ }^{18}$ No todos los enunciados interrogativos absolutos presentan este segmento, solo se realizó en 42 de los datos obtenidos; sin embargo no aparece en ninguno de los enunciados declarativos. También se presentaron dos casos en los que la vocal de la juntura terminal fue realizada con voz glotalizada (Frazier, 2009).

19 "A glottal stop is the sound (or, to be more exact, the lack of sound) that occurs when the vocal folds are held tightly together. [...] Glottal stops occur whenever one coughs" (Ladefoged y Johnson, 2011, p. 61); "A glottal stop is the sound (or lack of it) caused by bringing the vocal folds tightly together, cutting off all air from the lungs" (Ladefoged y Ferrari, 2012, p. 70).

20 "Vowels adjacent to phonemic glottal stop are often realized with a creaky voice quality immediately adjacent to the glottal closure" (Gordon, 2001, p. 1).
} 
Este segmento no se presenta de manera regular en todos los enunciados interrogativos colectados, ${ }^{21}$ sin embargo, no se encuentra en ningún caso de los enunciados declarativos, lo que parece indicar que podría contribuir en la identificación de los enunciados interrogativos. De acuerdo con Palancar (2009), en el otomí de San Ildefonso Tultepec se utiliza la marca ha para los enunciados interrogativos absolutos, de modo que para los hablantes otomíes este tipo de enunciados posee una marca de identificación, por tanto podría pensarse que, teniendo en cuenta que en el español no existe una marca diferente a la entonación para diferenciar entre enunciados declarativos e interrogativos absolutos, los hablantes bilingües puedan incluir un segmento como el cierre glotal que cumpla una función similar al del otomí, sin embargo, este caso amerita mayor indagación. ${ }^{22}$

Con todo, este fenómeno resulta particularmente interesante si se tiene en cuenta que esta unidad segmental no aparece en el inventario fonológico del español, pero sí hace parte del otomí (Palancar, 2009; Hekking y Andrés de Jesús, 1984)' y que además la distinción entre los enunciados declarativos e interrogativos absolutos en español ha sido una función prototípica de la entonación, sin embargo en esta variedad en contacto parece que una unidad segmental puede estar asociada (junto con otros factores acústicos) con una función distintiva que en español se ha considerado propia de las unidades suprasegmentales. ${ }^{23}$

${ }^{21} \mathrm{El}$ hecho de que este segmento glotal no se presente en todos los enunciados interrogativos absolutos (50\%) hace necesario un estudio exclusivo que considere otros tipos de enunciados interrogativos, pues no es descartable que, en algunos casos, los hablantes hayan generado una interrogativa de confirmación debido a la repetición del enunciado solicitado. De igual manera, es necesario considerar la obtención de datos con diferentes instrumentos para saber si esto incide en la inserción del segmento glotal; igualmente se debe incluir un mayor número de participantes que permita determinar si la dominancia de lengua afecta la presencia de este segmento. ${ }^{22}$ La presencia de este segmento glotal se encuentra de igual manera en variedades rurales del español hablado en el estado de Querétaro.

${ }^{23}$ Este es un aspecto que debe ser estudiado con más detenimiento, involucrando un mayor número de datos, específicamente reunidos para este fin, dado que es probable que su discontinuidad en los enunciados interrogativos absolutos pueda deberse a que los hablantes produjeran otros tipos de interrogación, pues en la conversación 


\section{Consideraciones finales}

En este documento se presentó una exploración inicial de la realización prosódica en los enunciados declarativos e interrogativos absolutos del español en hablantes bilingües español-otomí de San Ildefonso Tultepec. Los resultados preliminares permiten observar que existen bastantes similitudes entre ambos tipos de enunciados en esta variedad, como una tendencia a presentar junturas iniciales bajas \% $\mathrm{L}$ y un tono nuclear $\mathrm{L}+\mathrm{H}^{*}$ con finales bajos $\mathrm{L} \%$ y sostenidos M\%. El enunciado interrogativo, por su parte, presentó la inserción de un segmento glotal al final del enunciado que no ocurre en los enunciados declarativos, y que podría cumplir una función distintiva, aspecto que debe estudiarse con más detenimiento.

Con relación a la variedad en contacto, es posible trazar diferencias con la variedad del español metropolitano. En cuanto a los enunciados declarativos, estas diferencias son observables en el segmento prenuclear que, en el caso de los bilingües, presenta casos de realización plana mientras que en los hablantes de la Ciudad de México se presentaron picos desplazados. Con respecto a los enunciados interrogativos se observa que las hablantes bilingües tiene una configuración nuclear $\mathrm{L}+\mathrm{H}^{*} \mathrm{~L} \%$ seguido por la presencia de un segmento glotal que no se reportan en el español metropolitano. De acuerdo con lo anterior, la variedad en contacto cuenta con rasgos entonativos particulares que, en principio, permitirían considerarla como otra variedad del español, aunque se requiere de más estudios que involucren la entonación tanto del español regional como del otomí para reconocer posibles transferencias de la prosodia de esta lengua.

\section{Referencias}

Alvord, M. S. (2006). Spanish Intonation in Contact: The case of Miami Cuban Bilinguals (tesis inédita de doctorado). Universidad de Minnesota, Minneapolis, Estados Unidos de América.

espontánea con sus familiares también se inserta este segmento en interrogativas de confirmación; sin embargo, esta tarea excede los límites del presente estudio. 
Ávila, S. (2003). La entonación del enunciado interrogativo en el español de la Ciudad de México. En E. Herrera y P. Martín (eds.), La tonía: dimensiones fonéticas y fonológicas (pp. 331-355). México: El Colegio de México.

BArnes, H. y Michnowicz, J. (2013). Peak Alignment in Semispontaneus Bilingual Chipilo Spanish. En A. M. Carvalho y S. Beaudrie (eds.), Selected Proceedings of the 6th Workshop on Spanish Sociolinguistics (pp. 109-122). Cascadilla Proceedings Project. Recuperado de https://www.lingref. com/cpp/wss/6/paper2861.pdf.

Birdsong, D., Gertken, L. M. y Amengual, M. (2012). Bilingual Language Profile: An Easy-to-Use Instrument to Assess Bilingualism. Recuperado de https://sites.la.utexas. edu/bilingual/.

Boersma, P. y Weenink, D. (1992-2019). Praat: Doing Phonetics by Computer. Ámsterdam: Universidad de Amsterdam. Recuperado de http://www.fon.hum.uva.nl/praat/.

Braid, B. O. (2015). Pre-nuclear Peak Alignment in the Spanish of Spanish-K'ichee' (Mayan) Bilinguals. En E. Willis, P. Martín y E. Herrera Zendejas (eds.), Proceedings of Laboratory Approaches to Romance Phonology (LARP) 6 (pp. 163-174). Sommerviller, MA: Cascadilla Proceedings Project.

Braid, B. O. y Francisco, A. (2012). Realizaciones fonéticas de /V?/ en Q'anjob’al (Maya). En Proceedings of CILLA V. Austin: Universidad de Texas. Recuperado de https://ailla. utexas.org/sites/default/files/documents/Baird_Pascual_ CILLA_V.pdf.

Chamoreau, C. (2013). Diversidad lingüística en México. Amerindia, 37(1), 3-20.

Colantoni, L. (2011). Broad-Focus Declaratives in Argentine Spanish Contact and no Contact and non-Contact Varieties. En G. Christoph y L. Conxita (eds.), Intonational Phasing at the Interfaces: Cross-Linguistic and Bilingual Studies in Romance an Germanic (pp. 183-212). Ámsterdam: John Benjamins.

Coronado, J. (2014). Entonación rural del español de México. En P. Martín y L. Orozco (eds.), Argumentos cuantitavos 
y cualitativos en sociolingüística (pp. 61-78). México: El Colegio de México.

Dankel, P. y Soto, M. (2018). Patrones prosódicos en construcciones enumerativas: una visión desde la perspectiva del contacto español-quechua. Revista Internacional de Lingüística Iberoamericana, 16(32), 35-52. Recuperado de https: //edoc.unibas.ch/67260/.

De-la-Mota, C., Martín Butragueño, P. y Prieto, P. (2010). Mexican Spanish Intonation. En P. Prieto y P. Roseano (eds.), Transcription of Intonation of the Spanish Language (pp. 319-350). Múnich: Lincom Europa.

Dorta, J. (2019). Transferencia de la entonación y bilingüísmo: el caso de San Antonio, Texas. Estudios Filológicos, 63, 57179.

Elordieta, G. (2003). The Spanish Intonation of Speakers of a Basque Pich-Accent Dialect. Catalan Journal of Linguistics, 2, 67-95.

Estebas-Vilaplana, E. y Prieto, P. (2008). La notación prosódica del español: una revisión del Sp_ToBI. Estudios de Fonética Experimental, XVII, 263-283. Recuperado de https://core.ac.uk/download/pdf/39111168.pdf.

Frazier, M. (2009). The Production and Perception of Pitch and Glottalization in Yucatec Maya (tesis inédita de doctorado). Universidad de Carolina del Norte, Chaper Hill, Estados Unidos de América.

Frota, S. y Prieto, P. (2015). Intonation in Romance: Systemic Similarities and Differences. En S. Frota y P. Prieto (eds.), Intonation in Romance (pp. 392-418). Oxford: Orxfod University Press.

GIL, C. (2011). El español del municipio de la Paz, Baja California Sur: variación fónica y entonativa (tesis inédita de licenciatura). Escuela Nacional de Antropología e Historia, Ciudad de México, México.

Gordon, M. (2001). Linguistic Aspects of Voice Quality with Special Reference to Athabaskan. En S. Tuttle y G. Holton (eds.), Proceedings of the 2001 Athabaskan Languages Conference (pp. 163-178). Los Ángeles: University of California. 
Guerrero, A. (2015). Patrones tonales y acento en otomí. En E. Zendejas (ed.), Tono, acentos y estructuras métricas en lenguas mexicanas (pp. 235-260). México: El Colegio de México.

Gutiérrez-Bravo, R. (2008). La identificación de los tópicos y los focos. Nueva Revista de Filología Hispánica, 56, 363401. Recuperado de https://nrfh.colmex.mx/index.php/ nrfh/article/view/969.

Gussenhoven, C. (2004). The Phonology of Tone and Intonation. Cambridge: Cambridge University Press.

Hekking, E. y Andrés de Jesús, S. (1984). Gramática otomí. Querétaro: Universidad Autónoma de Querétaro.

Henriksen, N. y Fafulas, S. (2017). Prosodic Timing and Language Contact: Spanish and Yagua in Amazonian Peru. Studies in Hispanic and Lusophone Linguistics, 10(2), 225257. Recuperado de https://doi.org/10.1515/shll-2017-0007. Hualde, J. I. (2003). El modelo métrico y autosegmental. En

P. Prieto (ed.), Teorías de la entonación (pp. 155-184). Barcelona: Ariel.

Hualde, J. I. y Prieto, P. (2015) Intonational Variation in Spanish: European and American Varieties. En S. Frota y P. Prieto (eds.), Intonational Variation in Romance (pp. 350391). Oxford: Oxford University Press.

Instituto Nacional de Lenguas Indígenas (Inali) (2008). Catálogo de las lenguas indígenas nacionales: variantes lingüísticas de México con sus autodenominaciones $y$ referencias geoestadísticas. Recuperado de https://www. inali. gob. $\mathrm{mx} / \mathrm{clin}$-inali/.

Koкcmann, M.(s/f).EscuelaruralUruguayniños. Recuperadode https://www.pinterest.com.mx/pin/51932201923487751/.

LAdD, D. R. (1996). Intonational Phonology. Cambridge: Cambridge University Press.

Ladefoged, P. y Ferrari, S. (2012). Vowels and Consonants (3a ed.). Oxford: Blackwell Publishing.

Ladefoged, P. y Johnson, K. (2011). A Course in Phonetics (6 ed.). Boston: Wadsworth.

Martín Butragueño, P. (2004). Configuraciones circunflejas en la entonación del español mexicano. Revista de Filología Española, 84, 347-373. Dor: 10.3989/rfe.2004.v84.i2.111. 
Martín Butragueño, P. (2006). El estudio de la entonación del español de México. En M. Sedano, A. Bolívar y M. Shiro (comps.), Haciendo lingüística: homenaje a Paola Bentivoglio (pp. 105-126). Caracas: Universidad Central de Venezuela. Martín ButragueÑo, P. (2011). Estratificación sociolingüística de la entonación circunfleja mexicana. En P. Martín Butragueño (ed.), Realismo en el análisis de corpus orales. Primer Coloquio de Cambio y Variación Lingüística (pp. 9321). México: El Colegio de México.

Mendoza, É. (2014). La impresión de un tono: estudio sociolingüístico de la entonación de Cuapiaxtla, Tlaxcala (tesis inédita de doctorado). El Colegio de México, Ciudad de México, México. Recuperado de https://cell.colmex.mx/ index.php/alumnos/tesis/tesis-doctorado-linguistica.

Mendoza, É. (2019a). Entonación de los enunciados aseverativos en el Español de Tuxtla Gutiérrez, Chiapas. Nueva Revista de Filología Hispánica, LXVII(1), 41-76. DOI: 10. 24201/nrfh.v67i1.3464.

Mendoza, É. (2019b). Entonación de enunciados interrogativos en el español de Morelia, Michoacán. Anuario de Letras. Lingüística y Filología, 7(1), 157-198. DOI: 10.19130/iifl. adel.7.1.2019.1532.

Michnowicz, J. y Barnes, H. (2013). A Sociolinguistic Analysis of Pre-nuclear Peak Alignment in Yucatan Spanish. En C. Howe, S. E. Blackwell y M. Lubbers (eds.), Selected Proceedings of the 15th Hispanic Symposium (pp. 221-235). Sommerville, MA: Cascadilla Proceedings Project.

Montellano, U. (2017). Características prosódicas del español de Monterrey: enunciados declarativos neutros y enunciados interrogativos absolutos (tesis inédita de licenciatura). Escuela Nacional de Antropología e Historia, Ciudad de México, México.

Muntendam, A. (2012). Information Structure and Intonation in Andean Spanish. eLenguage. Recuperado de http:// elanguage.net/journals/lsameeting/issue/view/2012.

Muntendam, A. y Torreira, F. (2016). Focus and Prosody in Spanish and Quechua. En M. E. Armstrong, N. Henriksen y M. del M. Vanrell (eds.), Intonational Grammar in Ibero- 
Romance (pp. 70-89). Ámsterdam: John Benjamins. DoI: 10.1075/ihll.6.04mun.

Murrieta, L. (2016). Acercamiento al análisis experimental del umbral de percepción entonativa en el español del centro de México. Estudios de Lingüística Aplicada, 34(63), 153-166.

NAvA, E. F. (2010). El catálogo de las lenguas indígenas nacionales y la lingüística otopame. En A. M. Salazar y V. Kugel (eds.), Homenaje a Yolanda Lastra. X. Coloquio Internacional sobre Otopames. (pp. 81-122). México: Universidad Nacional Autónoma de México, Instituto Nacional de Lenguas Indígenas.

O'Rourke, E. (2012). The Realization of Contrastive Focus in Peruvian Spanish Intonation. Lingua, 122(5), 494-510.

Palancar, E. L. (2009). Gramática y textos del hñöñhö otomí de San Ildefonso Tultepec, Querétaro, vol. 1. México: Plaza y Valdés.

Pamies, A., Fernández, A. M., Martínez-Celdrán, E., OrTEgA, A. y Amorós, M. C. (2002). Umbrales tonales en español peninsular. En J. Díaz García (ed.), Actas del II Congreso Nacional de Fonética Experimental (pp. 272-278). Sevilla: Universidad de Sevilla.

Pierrehumbert, J. B. (1980). The Phonetics and Phonology of English Intonation (tesis inédita de doctorado). Massachussetts Institute of Technology, Cambridge, Estados Unidos de América. Recuperado de http://www.phon. ox.ac.uk/ jpierrehumbert/publications.html.

Prieto, P. (2003). Teorías lingüísticas de la entonación. Barcelona: Ariel.

Prieto, P. y Roseano, P. (2010). Transcription of Intonation of the Spanish Language. Múnich: Lincom Europa.

Rietveld, A. C. y Gussenhoven, C. (1985). On the Relation between Pitch Excusion Size and Prommence. Journal of Phonetics, 13, 299-308.

Rijswijk, R. van y Muntendam, A. (2014). The Prosody of Focus in the Spanish of Quechua-Spanish Bilinguals: a case Study on Noun Phrases. International Journal of Bilingualism, 18(6), 614-632.

Robles-Puente, S. (2012). Two Languages, Two Intonations? Statements and yes/no Questions in Spanish and Basque. 
En R. Campos Astorkiza y J. Franco (coords.), Papers in Linguistics by the BIDE Generation, Anuario del Seminario de Filología Vasca 'Julio de Urquijo', XLVI(1), 251-262.

Ruiz, M., Ulloa, O. y Chinuaicura, A. (2019). Acento y entonación en enunciados declarativos del español de Chile y mapudungun: primer acercamiento a la prosodia de ambas lenguas en contacto. Alpha, 49, 261-274. DoI: 10.32735/ S0718-2201201900049754.

Simonet, M. (2011). Intonational Convergence in Language Contact: Utterance-Final F0 Contours in Catalan Spanish Bilinguals. Journal of the International Phonetic Association, 41, 157-184.

Uth, M. y Gutiérrez-Bravo, R. (2020). Language Contact an Intonation: Evidence from Contrastive Focus Marking and Loanwords in Yucatecan Spanish and Yucatec Maya. Bulletin of Hispanic Studies, 97(1), 31-58.

Velázquez, E. P. (2016). Stability of Nahualt and Spanish Intonation Systems of Bilingual Nahualt Speakers from the Mexican Veracruz Huasteca Region. En Speech Prosody (pp. 1148-1152). DoI: 10.21437/SpeechProsody.2016-236. Zimmermann, K. (2001). El contacto de las lenguas amerindias con el español de México. Revista Internacional de Lingüística Iberoamericana, 2(4), 19-39. Recuperado de http:// red.pucp.edu.pe/ridei/files/2011/08/115.pdf. 